\section{Lesion-site-dependent responses to therapy after aphasic stroke}

\section{INTRODUCTION}

Stroke survivors with language difficulties (aphasia) vary: some recover quickly while others suffer long-term impairments, and different patients respond differently to the same speech and language therapies. ${ }^{1}$ In recent years, we and others have shown that much of the variability in language outcomes after stroke can be explained by reference to the details of the brain damage that individual patients have suffered. ${ }^{2}$ Here, we show that this same information can be used to predict responses of patients who had stroke to treatment.

\section{METHODS}

Detailed methods are provided in online supplementary material: here, we summarise the key points. Our focus is on a novel treatment for Central Alexia (CA): an acquired reading disorder in the context of a general language impairment (aphasia). Patients with CA are slow to read, make frequent errors and have additional problems with spoken and written language. Our intervention is a computerised therapy embodied in an application called 'iReadMore', which uses multimodal cueing and massed practice to improve patients' singleword reading skills. ${ }^{3}$

Our study included 23 participants with $\mathrm{CA}$ after left hemisphere stroke (see online supplementary table), recruited through both the PLORAS project ${ }^{2}$ and the outpatient speech and language therapy services at the National Hospital for Neurology and Neurosurgery, University College London Hospitals. Before the treatment began, each participant's cognitive skills were assessed with an extensive protocol including linguistic and non-linguistic tests, yielding a total of 28 pretreatment behavioural variables per patient. We also acquired structural MRI from each patient, extracting lesion images using the Automatic Lesion Identification toolbox. ${ }^{4}$ The outputs (binary lesion images) were encoded as the proportion that each lesion destroyed, of a series of 69 anatomically defined regions of interest. With four demographic variables (age at therapy onset, time since stroke, sex and lesion volume), we had a total of 101 pretreatment variables to consider; our dependent variable was absolute change in the participants' single-word reading skills.

We ran two analyses with these data. Our first, 'explanatory' (in-sample) analysis quantified the relative utility of: (1) behavioural and demographic data and (2) lesion location data, in explaining individual patients' responses to treatment (ie, improvement in single-word reading accuracy). We did this by fitting linear models using each set of variables, both separately and in combination, using the Automatic Linear Modelling (ALM) facility distributed with the SPSS software package. Our second, 'predictive' (out-of-sample) analysis asked whether the pretreatment data could be used to predict responses in new patients. We did this by embedding the ALM process within a cross-validation process.

\section{RESULTS}

Analysis 1: explanatory (in-sample) analysis

The Aikaike Information Criterion (AIC) for the model derived from behavioural and demographics data alone was higher (worse) than that selected from lesion data alone (81.75 vs 69.35$)$, and the model selected from all of the data together was better still $(\mathrm{AIC}=47.98)$. Bayes factors corresponding to these differences were 493 (behavioural and demographics vs lesions) and 43696, respectively (all data together vs lesions alone): that is, very strong evidence both (1) that lesion data explain more of the variance in the patients' treatment responses than the behavioural and demographic data alone and (2) that the combination of the data yields a better explanation than either set alone. Only the 'neuroimaging only' and 'combined data' models were significant, as assessed relative to null distributions of regression coefficients derived when the same models were regressed against 1000 permutations of the treatment responses (ie, actual $\mathrm{R}^{2}>95 \%$ of null statistics). 


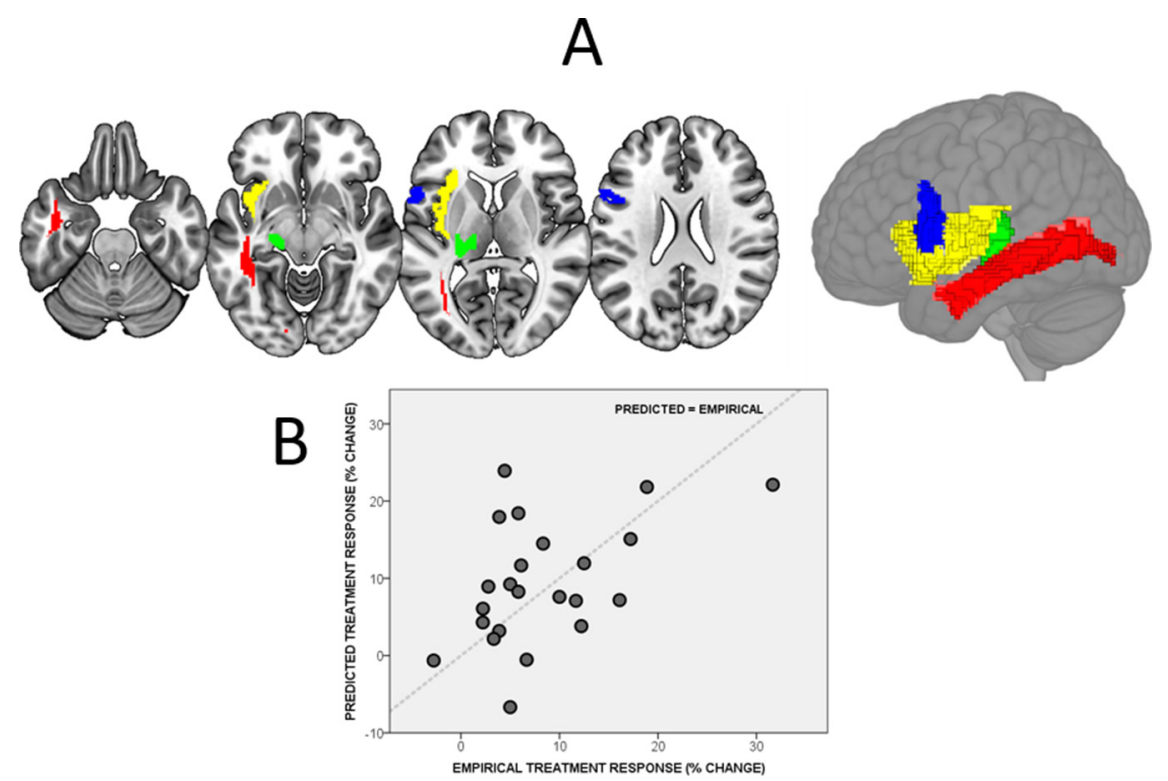

Figure 1 (A) The brain regions implicated in our combined model, displayed both on axial slices of the brain (left) at $Z=-24,-8,7$ and 23 and on a rendered whole brain (right). The regions are: (a) the white matter connecting the thalamus to the parietal cortex (green); (b) the inferior longitudinal fasciculus (red); (c) Broca's area 44 (blue) and (d) the left insula (yellow). (B) A scatter plot of predicted versus empirical treatment responses from our out-of-sample analysis. The dashed line is at $\mathrm{y}=\mathrm{x}$ : perfect predictions would fall along this line.

The best, explanatory model that we found included: (1) age at therapy onset; (2) accuracy in the Neale reading test; (3) comprehension in the Neale reading test; (4) accuracy in the written semantic matching task; (5) damage to the white matter connecting the thalamus to the parietal cortex and damage to left (6) Broca's area; (7) insula and (8) inferior longitudinal fasciculus (adjusted $\mathrm{R}^{2}=0.94$ ). Figure 1A displays the brain regions from this model.

\section{Analysis 2: predictive (out-of-sample) analysis}

Predicted treatment responses from the cross-validation analysis, using the combined demographic, behavioural and lesion location data, were significantly correlated with the patients' empirical treatment responses $(\mathrm{r}=0.48,95 \% \mathrm{CI}$ 0.08 , to $0.75, \mathrm{p}=0.02)$; see figure $1 \mathrm{~B}$. Predictions driven by models using either demographic and behavioural data or lesion location data, separately, were not significantly correlated with the empirical treatment responses (both $\mathrm{p}>0.1$ ).

\section{DISCUSSION}

Past studies have suggested that responses to treatment for word-finding deficits (anomia) after stroke may be related to pretreatment behavioural skills ${ }^{5}$ or lesions. $^{6} 7$ Our results are consistent with that work and also extend it by: (1) quantifying the relative value of pretreatment structural MRI versus demographic and behavioural data, in explaining treatment responses and (2) demonstrating that, in combination (though not separately), these pretreatment data can be used to predict new patients' responses to treatment.

We have only considered a single therapy (iReadMore), focused on a specific aphasic deficit (CA), in a study with specific inclusion criteria at both the lesion level and the behavioural level (see online supplementary material). And though large by the standards of other similar therapy studies, our sample is still too small either to properly constrain multivariable models or to measure their predictive power precisely. So our results are necessarily preliminary.

However, they are also plausible. Cognitive neuroanatomy is specialised: the lesion damage that patients have suffered should determine both their initial symptoms and their likely potential to recover. Our best model of those treatment responses is also reasonable. Initial reading accuracy is naturally relevant to patients' likely gains after reading therapy, and preserved semantic skills have been associated with recovery from aphasia. ${ }^{8}$ The inferior longitudinal fasciculus, the insula and Broca's area have all been associated with reading in neuroimaging experiments, ${ }^{9-12}$ and it is at least plausible that older patients responded less well to our treatment. Finally, our predictive analysis should penalise small samples, because these will tend to maximise the variability between folds of the cross-validation process. Indeed, our results demonstrate the gulf that separates prediction from in-sample analysis: we could 'explain' 94\% of the variance in those treatment responses, but predict only 23\%. The out-of-sample effect size should grow with increasing sample size, but the in-sample effect size is clearly inflated.

In any case, we hope that these results will encourage further attempts to characterise lesion-site-dependent treatment effects and to distinguish predictable variance from noise in this area-and that further results like this will drive the development of a more personalised medicine for stroke survivors with aphasia.

Oscar M Aguilar, ${ }^{1,2,3}$ Sheila J Kerry, ${ }^{4}$ Yean-Hoon Ong, ${ }^{2}$ Martina F Callaghan, ${ }^{2}$ Jennifer Crinion,

Zoe Victoria Joan Woodhead, ${ }^{1,2,5}$ Cathy J Price, ${ }^{2}$ Alexander P Leff, ${ }^{1,2,4}$ Thomas M H Hope ${ }^{2}$

'Department of Brain Repair and Rehabilitation, University College London, London, UK

${ }^{2}$ Wellcome Centre for Human Neuroimaging, University College London, London, UK

${ }^{3}$ Facultad de Psicologia, Pontificia Universidad

Javeriana, Bogota, Colombia

${ }^{4}$ Institute of Cognitive Neuroscience, University College London, London, UK

${ }^{5}$ Department of Experimental Psychology, University of Oxford, Oxford, UK

Correspondence to Dr Thomas M H Hope, Wellcome Centre for Human Neuroimaging, University College London, London WC1N 3BG, UK ; t.hope@ucl.ac.uk

Contributors APL, JC, ZVJW, MFC and TMHH: designed research. APL and CJP: funding acquisition. OMA and TMHH: performed research. APL and $J C$ : handled patient recruitment. Y-HO: software development. OMA, TMHH, SJK and ZVJW: analysed data. OMA and TMHH: wrote the paper.

Funding This study was supported by the Medical Research Council (MR/K022563/11, MR/M023672/1), Wellcome (091593/Z/10/Z, 097720/Z/11/Z and 205103/Z/16/Z), the COLCIENCIAS Administrative Department of Science, Technology and Innovation (programme 529, 2011) and the Stroke Association (TSA PDF 2017/02)

Disclaimer The funders had no participation in the design and results of this study.

Competing interests None declared.

Patient consent Not required.

Ethics approval London Queen Square Research Ethics Committee at University College London.

Provenance and peer review Not commissioned; externally peer reviewed.

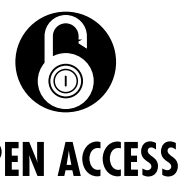

Open access This is an open access article distributed in accordance with the terms of the Creative Commons Attribution (CC BY 4.0) license, which permits others to distribute, remix, adapt and build upon this work, for 
commercial use, provided the original work is properly cited. See: http://creativecommons.org/licenses/by/4.0/

(c) Article author(s) (or their employer(s) unless otherwise stated in the text of the article) 2018. All rights reserved. No commercial use is permitted unless otherwise expressly granted.

\section{Check for updates}

To cite Aguilar OM, Kerry SJ, Ong Y-H, et al. J Neurol Neurosurg Psychiatry 2018;89:1352-1354.

Received 13 October 2017

Revised 15 February 2018

Accepted 26 February 2018

Published Online First 17 April 2018

I Neurol Neurosurg Psychiatry 2018;89:1352-1354. doi:10.1136/jnnp-2017-317446

\section{REFERENCES}

1 Brady MC, Kelly H, Godwin J, et al. Speech and language therapy for aphasia following stroke. Cochrane Database Syst Rev 2016:6:CD000425

2 Seghier ML, Patel E, Prejawa S, et al. The PLORAS Database: A data repository for Predicting Language Outcome and Recovery After Stroke. Neuroimage 2016;124:1208-12.

3 Woodhead ZV, Penny W, Barnes GR, et al. Reading therapy strengthens top-down connectivity in patients with pure alexia. Brain 2013;136:2579-91.

4 Seghier ML, Ramlackhansingh A, Crinion J, et al. Lesion identification using unified segmentationnormalisation models and fuzzy clustering. Neuroimage 2008;41:1253-66.

5 Lambon Ralph MA, Snell C, Fillingham JK, et al. Predicting the outcome of anomia therapy for people with aphasia post CVA: both language and cognitive status are key predictors. Neuropsychol Rehabil 2010:20:289-305.

6 Naeser MA, Baker EH, Palumbo CL, et al. Lesion site patterns in severe, nonverbal aphasia to predict outcome with a computer-assisted treatment program. Arch Neurol 1998;55:1438-48.
7 Fridriksson J. Preservation and modulation of specific left hemisphere regions is vital for treated recovery from anomia in stroke. J Neurosci 2010;30:11558-64.

8 Fucetola R, Connor LT, Perry J, et al. Aphasia severity, semantics, and depression predict functional communication in acquired aphasia. Aphasiology 2006:20:449-61.

9 Broca P. Remarques sur le siége de la faculté du langage articulé, suivies d'une observation d'aphémie (perte de la parole). Bulletin de la Société Anatomique 1861:6:330-57.

10 Klein M, Grainger J, Wheat KL, et al. Early Activity in Broca's Area During Reading Reflects Fast Access to Articulatory Codes From Print. Cereb Cortex 2015:25:1715-23.

11 Dronkers NF. A new brain region for coordinating speech articulation. Nature 1996:384:159-61.

12 Ripamonti E, Aggujaro S, Molteni F, et al. The anatomical foundations of acquired reading disorders: a neuropsychological verification of the dual-route model of reading. Brain Lang 2014;134:44-67. 